\title{
Efeitos de fatores comportamentais no desempenho da tarefa de peritos contábeis judiciais
}

\author{
Alexandre Corrêa dos Santos \\ https://orcid.org/0000-0003-3119-1586 \\ Ilse Maria Beuren \\ https://orcid.org/0000-0003-4007-6408
}

\section{Resumo}

Objetivo: Analisar os efeitos do estilo de tomada de decisão, da flexibilidade cognitiva e dos traços de personalidade no desempenho da tarefa de peritos contábeis judiciais.

Método: Uma survey foi realizada com peritos contábeis judiciais, o que resultou em uma amostra final de 259 respostas válidas. Para a análise dos dados, utilizou-se a técnica de modelagem de equações estruturais por mínimos quadrados parciais.

Resultados: Os fatores comportamentais flexibilidade cognitiva e traços de personalidade apresentaram relação positiva com o desempenho da tarefa, enquanto o fator estilos de tomada de decisão apresentou relação negativa.

Contribuições: Esses resultados sinalizam que é preciso expandir o escopo de análise do desempenho da tarefa para além da perspectiva positivista, ou seja, não se pode presumir que um indivíduo melhore seu desempenho unicamente por comandos racionais de trabalho. Abrem caminhos de investigação e incentivam a busca de novas abordagens e constructos para explicar os comportamentos relacionados ao desempenho da tarefa de indivíduos.

Palavras-chave: Estilos de tomada de decisão; Flexibilidade cognitiva; Traços de personalidade; Peritos contábeis judiciais. 


\section{Introdução}

A racionalidade do processo decisório é abordada na literatura sob duas perspectivas principais. A primeira investiga a decisão sob a ótica racional (Melo \& Fucidji, 2016) em uma perspectiva econômica de utilidade esperada (Neumann \& Morgenstern, 1947; Malkiel \& Fama, 1970; Santos \& Barros, 2011). A segunda, a partir da ideia de racionalidade limitada (Simon, 1955), considera o debate dos fatores comportamentais. Embora coexistentes, de um lado, tem-se a visão econômica voltada para os meios, instrumentos e processos formais que os indivíduos utilizam para tomar decisões ótimas; de outro lado, a perspectiva psicológica analisa as decisões humanas a partir da influência da mente e do comportamento, com foco na motivação, cognição, atitudes, ações e comunicação (Birnberg, Luft \& Shields, 2006).

Portanto, o processo decisório, mais do que a seleção e a avaliação de alternativas sob a perspectiva econômica, representa a maneira como os sujeitos captam a informação para a decisão e como eles analisam o problema envolvido e os julgamentos que formam para fazer uma escolha (Perez, Valero, Cesar \& Medeiros Júnior, 2017). Essa visão mais abrangente é objeto de estudo da psicologia cognitiva, que se ocupa da investigação das percepções do indivíduo em relação ao mundo exterior, onde a realidade externa é construída a partir da visão interior, por meio das representações (Neufeld, Brust \& Stein, 2011). Todavia, a psicologia também trata o processo decisório a partir de sua subárea que aborda os traços de personalidade, que são características relativamente estáveis dos indivíduos, que podem afetar a percepção de risco nas tarefas (Lauriola \& Levin, 2001).

Embora a psicologia tenha alcançado um nível de conhecimento satisfatório nas duas abordagens apresentadas, há lacunas ligadas a questões sobre o processo de tomada de decisão, como, por exemplo, a mudança dos traços de personalidade pela vontade (Hudson \& Fraley, 2015), a mudança de personalidade ao longo da vida (Chopik \& Kitayama, 2017) e questões relativas às limitações cognitivas (Cagnin, 2009). Diante disso, pressupõe-se que o desempenho de tarefas pode ser analisado sob uma perspectiva holística, em que capacidades cognitivas e traços de personalidade interagem e influenciam as escolhas (Macêdo, 2016).

Os estilos de tomada de decisão referem-se às diferenças entre as pessoas quanto ao processamento da informação que será utilizado para solucionar problemas e tomar decisões em diferentes contextos (Thunholm, 2004), sob a ótica particular de reação dos indivíduos ao contexto e como ele é interpretado (Scott \& Bruce, 1995). Esta variável ajuda a compreender diferenças individuais que influenciam a decisão (Benbasat \& Dexter, 1982). É utilizada pela literatura para analisar o efeito de estilos cognitivos nas decisões de alocação de recursos (Chenhall \& Morris, 1991), na solução de problemas complexos de negócios por meio de informações contábeis (Cheng, Luckett \& Schulz, 2003) e como fator determinante das escolhas contábeis da organização (Ge, Matsumoto \& Zhang, 2011).

Dada a capacidade de os indivíduos racionalizar e processar informações, este estudo também explora o fator flexibilidade cognitiva. Segundo Martin e Rubin (1995), a flexibilidade cognitiva refere-se à consciência individual, a qual, diante de qualquer situação em que haja alternativas disponíveis, abre a disposição para a flexibilidade e adaptação para a situação, apoiada na autoeficácia em ser flexível.

Quanto aos traços de personalidade, pessoas com baixa estabilidade emocional (neuroticismo) são mais suscetíveis a emoções negativas e inibição de recursos cognitivos, geralmente projetam suas emoções internas em seu trabalho (George \& Zhou, 2007). O traço conscienciosidade afeta o desempenho da tarefa por meio de mecanismos motivacionais, como na definição de grandes objetivos (Barrick \& Mount, 1993; Monzani, Ripoll \& Peiró, 2015).

Cubel, Nuevo-Chiquero, Sanchez-Pages e Fernandez (2016), ao analisarem os cinco macrotraços de personalidade (abertura à experiência, conscienciosidade, extroversão, neuroticismo e amabilidade), encontraram que sujeitos mais neuróticos apresentam pior desempenho de produtividade, e que indivíduos mais conscientes apresentam melhor desempenho. Nesta perspectiva, o desempenho da tarefa é tomado como o resultado de comportamentos que refletem em ações (Bendassolli, 2012) e está inserido em um processo coletivo de construção de significados (Weick, 1995), portanto, vai além da função, depende de interações constantes com pares e stakeholders em ambientes incertos. 
Na pesquisa contábil, além do mainstream estar voltado à visão funcionalista dos fenômenos, há certa assimetria dos achados entre abordagens decisórias comportamentais, ora em estudos cognitivos (Lucena, Fernandes \& Silva, 2011; Butler \& Ghosh, 2015, Oblak, Licen \& Slapnicar, 2018), ora em estudos da personalidade (Monzani, Ripoll \& Peiró, 2015). Esse aspecto instiga mais pesquisas, além da carência de estudos com uma abordagem mais holística sobre o comportamento decisório e seu efeito prático, bem como a utilização de instrumentos que consigam captar diferentes efeitos. Bromwich e Scapens (2016) advertem que pesquisadores que desejam um maior impacto sobre a prática, precisam estar cientes do que ocorre no mundo prático e, a partir disso, trabalhar em função de resolver seus problemas.

Nessa direção, os estilos de tomada de decisão, a flexibilidade cognitiva e os traços de personalidade são uma interessante via conjunta para investigar o desempenho da tarefa dos peritos contábeis, uma vez que influenciam o processamento de informações na fase de desenvolvimento de habilidades e conhecimentos (Riding, 1997). Assim, este estudo objetiva analisar os efeitos dos estilos de tomada de decisão, da flexibilidade cognitiva e dos traços de personalidade no desempenho da tarefa de peritos contábeis judiciais.

Analisar os estilos de tomada de decisão sob a ótica do desempenho da tarefa fornece meios para alocação de contadores em funções mais adaptativas às suas características cognitivas, de forma a melhorar o desempenho da tarefa (Fuller \& Kaplan, 2004). Diante dos desafios da tarefa do perito contábil, Lonescu (2012) explica que a flexibilidade cognitiva é uma característica-chave, que auxilia os indivíduos a realizar tarefas complexas, multitarefas, busca de novas soluções e adaptação à mudança de demandas. Nesta linha, as decisões dos profissionais peritos contábeis são o produto das escolhas sobre como desenvolver as rotinas planejadas em um trabalho (Marras, 2011). Segundo Mahama e Cheng (2013), o desempenho da tarefa pode ser explicado pela psicologia cognitiva, pois reflete as percepções individuais.

Este estudo contribui com a literatura ao considerar uma abordagem holística de análise dos efeitos dos estilos de tomada de decisão, da flexibilidade cognitiva e dos traços de personalidade no desempenho da tarefa de peritos contábeis judiciais. Nessa perspectiva, Santos, Sisto e Martins (2003) enfatizam que há diferenças na forma como as pessoas comumente pensam e sentem situações com as quais se defrontam, há tendências diferenciadas nas formas de aprender e relacionar os dados da realidade e de elaborar conclusões sobre eles. Além disso, avança nas pesquisas do campo da perícia contábil, cujo interesse tem se concentrado em temáticas de conformidade do trabalho pericial.

Dado que o trabalho pericial é desenvolvido em duas dimensões que não podem ser separadas, a técnica e a humana, analisar fatores comportamentais agrega à prática da área, uma vez que o trabalho de peritos contábeis é desenvolvido em um ambiente de interação com as partes processuais, assistentes técnicos e representantes do poder judiciário. As decisões da tarefa dos peritos contábeis são tomadas após todas as ponderações de assuntos que envolvem esses sujeitos e seus interesses, portanto, é possível pressupor que variáveis comportamentais complementam a racionalidade e influenciem a decisão da tarefa (Kahneman, 2012).

$\mathrm{Na}$ dimensão desempenho de tarefas de peritos contábeis, os resultados deste estudo sinalizam para órgãos reguladores a necessidade de criação de normas que considerem aspectos críticos de tomada de decisão do perito. Também podem estimular universidades a melhorarem a ementa da disciplina de perícia contábil, já que há um nível alto de desinformação sobre os desafios e oportunidades do mercado de trabalho do perito contábil (França \& Barbosa, 2015). Por fim, podem incentivar profissionais a buscar novos meios de formação para o trabalho, não limitados a atuação técnico-instrumental (Hoog, 2008). Assim, na direção do estudo de Murro e Beuren (2016) sobre formação de redes na perícia contábil, amplia-se o conhecimento teórico dessa área eminentemente pragmática e técnica. 


\section{Revisão da Literatura e Hipóteses}

O ser humano constantemente precisa tomar decisões em situações de risco ou incerteza, em que busca minimizar as possibilidades de resultados indesejados e potencializar resultados positivos desejados (Hastie, 2001). Para atingir os melhores resultados, a teoria econômica apoia-se na decisão racional, que pressupõe que os métodos formais são os meios mais eficazes de reduzir vieses e atingir objetivos (Lima Filho, Bruni, Sampaio, Cordeiro Filho \& Carvalho Jr., 2010). Bernstein (1997) considera a racionalidade essencial para a tomada de decisão sob incerteza, pois o comportamento racional torna as pessoas mais objetivas quanto às informações, sem os efeitos de tendências otimistas ou pessimistas.

No entanto, ao contrário do que propõem as teorias de escolhas racionais, oriundas das ciências econômicas, os decisores não têm domínio integral da racionalidade (Plous, 1993) e, nessas circunstâncias, os estilos cognitivos refletem diferenças individuais na organização cognitiva do indivíduo e os vê como elemento mediador entre a habilidade e a personalidade (Messick, 1984). Santos, Sisto e Martins (2003) consideram que os estilos cognitivos podem ajudar a explicar o limite entre a cognição e a personalidade. A racionalidade não supre o conhecimento fragmentado das condições que cercam a ação do homem e sua percepção dos fenômenos e das leis que possibilitam predizer as consequências futuras de suas ações com base no conhecimento das circunstâncias atuais (Pereira, Löbler \& Simonetto, 2010).

A psicologia se apoia na ideia de que o indivíduo desenvolve sua própria visão de mundo, logo o que pensa e o que sente pode ser uma percepção diferente diante das situações com as quais se defronta (Santos, Sisto \& Martins, 2003) e, dado o volume de informações e o ambiente complexo do trabalho (Luft, Shields, \& Thomas, 2016), as características pessoais do indivíduo influenciam seu estilo de decisão (Frankovsky, Birknerova \& Zbihlejova, 2016).

Quanto aos estilos de tomada de decisão, Scott e Bruce (1995) construíram, a partir da teoria, um instrumento denominado General Decision-Making Style Inventory (GDMS), que foi validado no Brasil por Löbler, Reis, Nishi e Tagliapietra (2019). Esse instrumento tem a função de capturar cada um dos cinco estilos de tomada de decisão (dependente, procrastinador, racional, intuitivo e espontâneo) e sua versão para o contexto brasileiro apresentou qualidades psicométricas adequadas e satisfatórias.

Por sua vez, o desempenho da tarefa, segundo Motowildo, Borman e Schmit (2013), é relacionado às atividades produtivas de transformação de matéria-prima em produtos e serviços, ou ainda aos serviços que atendem e mantêm o núcleo técnico. Portanto, essa variável tem relação direta com questões técnicas da organização, quer pela execução de processos técnicos ou pela manutenção dos requisitos técnicos.

A partir disso, no âmbito específico de desempenho da tarefa de peritos contábeis judiciais, formulase a primeira hipótese da pesquisa:

$\mathbf{H}_{1}$ : Os estilos de tomada de decisão influenciam positivamente o desempenho da tarefa de peritos contábeis judiciais.

Para além dos estilos de tomada de decisão, Pereira, Silva e Tavares Jr (2017) explicam que, em um ambiente influenciado pelas emoções, falta de conhecimento apropriado das situações e racionalidade limitada, as decisões contábeis são influenciadas por pressões dos stakeholders envolvidos. Esse cenário não é diferente no trabalho do perito, que se depara com conflitos de interesses das partes, debates metodológicos e pressão de prazo, portanto, faz escolhas e realiza julgamentos (Mendonça Neto, Cardoso, Oyadomari \& Silva, 2009). 
Nas diligências in loco e outros processos de socialização, o perito contábil interage com outros indivíduos e debate sobre seus interesses e sentimentos. Nessas circunstâncias, o comportamento passa pelo processo de cognição social, que é a tomada de consciência de alternativas, em que o nível de reconhecimento diferencia pessoas mais cognitivamente flexíveis e dispostas a ajustes daquelas que veem apenas uma resposta comportamental (Martin \& Rubin, 1995). Isto pode ser explicado pela dimensão adaptativa da teoria da flexibilidade cognitiva, pois o indivíduo pode produzir mudanças de forma a responder às exigências impostas pelas alterações dos problemas e situações (Guerra, Candeias \& Prieto, 2014). Assim, a forma de interpretação do ambiente e como ele altera o modelo mental para orientar o comportamento é influenciado pelo estilo cognitivo (Mueller \& Shepherd, 2016).

Essa interação que modifica e é modificada pelo ambiente está associada a componentes cognitivos chamados funções executivas e relaciona-se à capacidade de o indivíduo engajar-se em comportamentos orientados para objetivos, por meio de ações voluntárias, independentes, autônomas, auto-organizadas e orientada para metas (Capovilla, Assef \& Cozza, 2007). Essas funções estão ligadas ao planejamento, monitoramento e flexibilidade cognitiva, ao passo que a flexibilidade cognitiva influencia o comportamento para objetivos (Capovilla et al., 2007), portanto, é possível que afete o desempenho da tarefa.

A flexibilidade cognitiva, estudada inicialmente por Spiro, Vispoel, Schmitz, Samarapungavan e Boerger (1987), relaciona-se com níveis avançados de aquisição de conhecimento, o que possibilita agir com mais eficácia diante de situações novas ou complexas. Martínez e Brussoni (2018) analisaram tomadores de decisão especializados e concluíram que a flexibilidade cognitiva, ou seja, a capacidade de combinar o tipo de processamento cognitivo com o tipo de problema em questão permite que obtenham um desempenho melhor, pois trocam os hábitos decisórios por uma análise prévia mais profunda do contexto.

Nesse contexto, a flexibilidade cognitiva pode ser representada pela flexibilidade de atenção, um processo que envolve habilidades individuais de focalizar, alocar e refinar os estímulos; pela flexibilidade de representação, relacionada à capacidade de análise, síntese e recuperação da informação; e a flexibilidade de resposta, ligada à habilidade de criar estratégias, planos e programas, enfim habilidade ligada à decisão e execução (Guerra, 2013).

A partir disso, considerando o contexto particular de desempenho da tarefa de peritos contábeis judiciais, formula-se a segunda hipótese da pesquisa:

$\mathbf{H}_{2}$ : A flexibilidade cognitiva influencia positivamente o desempenho da tarefa de peritos contábeis judiciais.

Esta hipótese, caso confirmada, reforçará a ideia de que a competência técnica dos peritos contábeis judiciais é influenciada por fatores comportamentais cognitivos, o que permite pressupor que os níveis de atuação profissional são dependentes de características pessoais que diferenciam cada indivíduo e suas habilidades.

Contudo, é possível que além dos estilos de tomada de decisão e da flexibilidade cognitiva, paralelamente os traços de personalidade afetem o juízo sobre os fatos a serem decididos (Cunha, Silva, Peyerl \& Haveroth, 2019). A personalidade como característica dos indivíduos, sendo única e o distinguindo dos demais a partir de padrões consistentes de sentimentos, pensamentos e comportamentos (Trentini, Hutz, Bandeira, Teixeira, Gonçalves \& Thomazoni 2009), tende a influenciar os hábitos de reagir de uma forma particular em contextos de decisão (Scott \& Bruce, 1995).

Na literatura, Saucier (1994) explica que a consciência está ligada à capacidade de reflexão em relação aos fenômenos antes da tomada de decisão, a extroversão está associada ao nível de sociabilidade, a agradabilidade concerne à capacidade de ser agradável consigo e com os demais, o neuroticismo referese a um estado de maior tendência a desequilíbrios emocionais e, por fim, a abertura a experiências remete à aptidão a novos desafios. 
Segundo Debusscher, Hofmans e Fruit (2016), a relação entre os traços de personalidade e o de desempenho da tarefa ocorre por meio das percepções da pressão do trabalho e da complexidade da tarefa, que podem desencadear estados momentâneos de neuroticismo, que, por sua vez, predizem o desempenho momentâneo da tarefa. Nesta perspectiva, mas estreitando a lente para o contexto específico de desempenho da tarefa de peritos contábeis judiciais, formula-se a terceira hipótese da pesquisa:

$\mathbf{H}_{3}$ : Os traços de personalidade influenciam positivamente o desempenho da tarefa de peritos contábeis judiciais.

Na Figura 1 são apresentadas as três variáveis latentes (estilos de tomada de decisão, flexibilidade cognitiva e traços de personalidade), as duas primeiras são de ordem cognitiva, já os traços de personalidade são intrínsecos ao indivíduo.

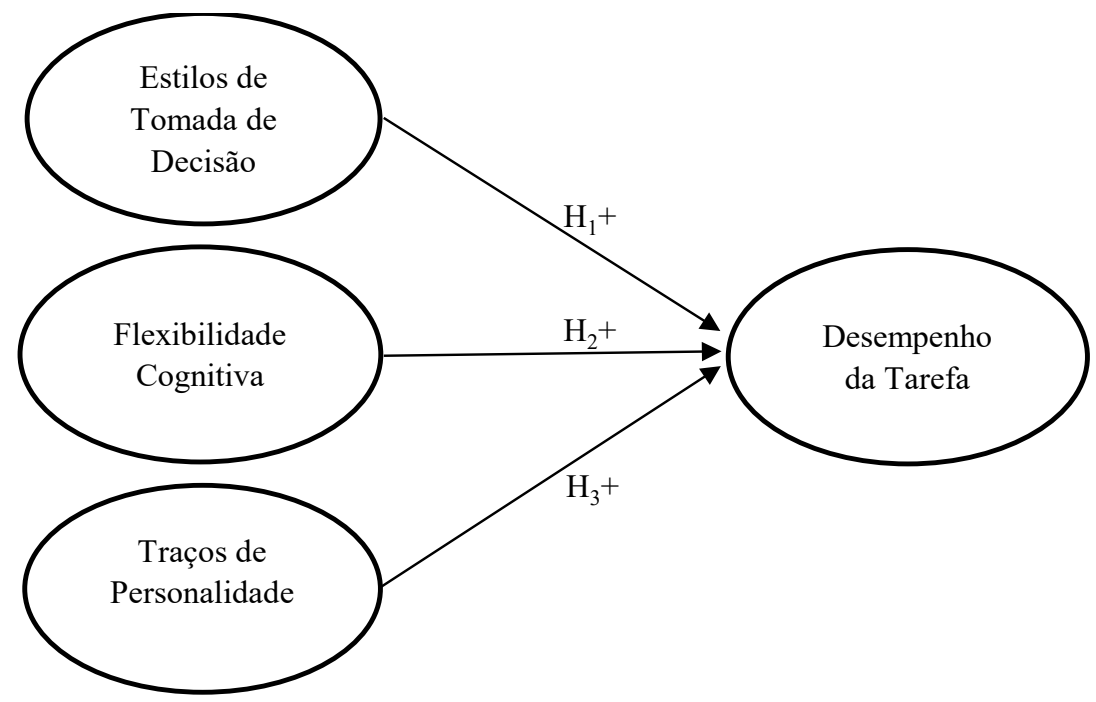

Figura 1. Modelo teórico da pesquisa

Fonte: elaboração própria.

Nas relações demonstradas, espera-se que o desempenho da tarefa seja compreendido a partir das variáveis propostas e que a literatura atual possa avançar por meio da análise conjunta de variáveis cognitivas e de traços de personalidade.

\section{Procedimentos Metodológicos}

Uma survey foi realizada tendo como população os peritos contábeis. As listas de peritos contábeis foram extraídas do Cadastro Nacional de Peritos do Conselho Federal de Contabilidade (CNPC, 2020) e das associações de profissionais de peritos. Definida a população, no período de 9 de novembro a 18 de dezembro de 2020, um questionário estruturado foi enviado, via e-mail, pela plataforma Google Forms $\odot$, para 3.200 peritos contábeis. A amostra final obtida foi de 259 respondentes, o que corresponde a 8,09\%.

O perfil dos respondentes aponta que $71,4 \%$ são do gênero masculino, $37,5 \%$ estão na faixa etária acima de 55 anos. Quanto ao nível de escolaridade, 62,9\% dos respondentes possuem pós-graduação em nível de especialização. Em relação ao tempo de atuação como perito contábil, chama a atenção que, apesar da faixa etária prevalente não ser de jovens, 30,9\% dos respondentes estão entre 1 a 5 anos atuando na área. Os dados demográficos descritos sugerem que os respondentes reúnem condições que lhes permitem responder o instrumento de pesquisa. 
Os instrumentos de pesquisa (Apêndice A) utilizados foram extraídos de estudos prévios que investigaram um ou mais constructos objeto desta investigação. A escala Likert de cinco pontos foi adotada para todos os blocos de assertivas, independentemente da escala original, porém, mantida a semântica inicial dos limites para cada instrumento.

Para capturar os estilos de tomada de decisão, foi utilizado o instrumento desenvolvido por Scott e Bruce (1995), denominado General Decision-Making Style Inventory (GDMS), que captura cada um dos cinco estilos de decisão (dependente, procrastinador, racional, intuitivo e espontâneo). O instrumento original apresenta 25 assertivas em escala Likert de cinco pontos, porém, utilizou-se o questionário validado no contexto brasileiro por Löbler, Reis, Nish e Tagliapietra (2019), que reduziu o número de assertivas para 19 e resultou em consistência interna (alfa de Cronbach) de 0,624.

A flexibilidade cognitiva foi investigada por meio do instrumento de pesquisa do estudo de Martin e Rubin (1995), que objetivou mensurar a capacidade de o indivíduo integrar conhecimento e ações relacionados à solução de problemas. Possui quatro assertivas para cada uma das três dimensões de flexibilidade (atenção, representação e resposta), originalmente em escala tipo Likert de seis pontos, variando entre 1 (discordo totalmente) a 6 (concordo totalmente), mas no presente estudo optou-se pela escala Likert de 5 pontos.

Para analisar os traços de personalidade, foi aplicado o instrumento de pesquisa do estudo de Gosling, Rentfrom e Swann (2003), que modelaram seu questionário nos descritores de instrumentos bigfive proposto por Goldberg (1992). O instrumento captura os cinco principais traços de personalidade consolidados na literatura e compreende dez questões em escala tipo Likert de sete pontos, duas questões para cada dimensão. Nesse estudo, convencionou-se utilizar a escala Likert de cinco pontos.

Por último, o desempenho da tarefa foi aferido a partir do instrumento de pesquisa idealizado por Kathuria e Davis (2001) e adaptado por Mahama e Cheng (2013), composto originalmente de quatro assertivas em escala Likert de cinco pontos, que variam entre 1 (não satisfeito) a 5 (muito satisfeito).

Para as estatísticas descritivas e teste das hipóteses, foi aplicada a técnica de modelagem de equações estruturais por mínimos quadrados parciais (PLS-SEM), por meio do software Smart PLS, versão 3. Ela pode ser definida como um conjunto de técnicas que ajudam a examinar simultaneamente um conjunto de relacionamentos teóricos entre uma ou mais variáveis independentes e uma ou mais variáveis dependentes (Hair Jr., Hult, Ringle \& Sarstedt, 2014). É adequada para modelar relações complexas com múltiplos relacionamentos de dependência e independência entre variáveis latentes (Hair Jr. et al., 2014).

\section{Descrição e Análise dos Resultados}

\subsection{Modelo de Mensuração}

Na modelagem de equações estruturais, os dados são analisados em duas etapas. Na primeira, realiza-se uma análise fatorial confirmatória para se observar a covariância entre as variáveis latentes, 0 que resulta no modelo de mensuração (Anderson \& Gerbing, 1988; Bido \& Silva, 2019). A segunda etapa da análise consiste no modelo estrutural, que reflete os ajustes necessários à otimização do modelo.

No modelo de mensuração, analisou-se a confiabilidade do modelo e a validade discriminante. A análise da validade discriminante deu-se a partir da verificação das cargas cruzadas, que apontam se os valores das cargas maiores das variáveis latentes originais são maiores do que as demais (Chin, 1998). Também foi observada a partir do critério de Fornell e Larcker (1981), ao verificar se as raízes quadradas dos valores da variância média extraída (AVE) de cada constructo são maiores do que as correlações dos constructos. 
Na Tabela 1, mostram-se os valores estatísticos das variáveis consideradas na análise da robustez do modelo, como o alfa de Cronbach, o rho_A, a confiabilidade composta, o $\mathrm{R}^{2}$ e a variância média (AVE).

Tabela 1

Modelo de mensuração

\begin{tabular}{|c|c|c|c|c|c|}
\hline \multicolumn{6}{|c|}{ Painel A - Sem exclusão de indicadores } \\
\hline Variáveis & $\begin{array}{l}\text { Alfa de } \\
\text { Cronbach }\end{array}$ & rho_A & $\begin{array}{l}\text { Confiabilidade } \\
\text { composta }\end{array}$ & $\mathbf{R}^{\mathbf{2}}$ & $\begin{array}{c}\text { Variância média } \\
\text { extraída (AVE) }\end{array}$ \\
\hline Estilos de tomada de decisão & 0,712 & 0,785 & 0,112 & 0,187 & 0,195 \\
\hline Flexibilidade cognitiva & 0,730 & 0,759 & 0,792 & 0,159 & 0,252 \\
\hline Traços de personalidade & 0,681 & 0,718 & 0,744 & 0,326 & 0,252 \\
\hline Desempenho da tarefa & 0,786 & 0,813 & 0,862 & 0,327 & 0,613 \\
\hline \multicolumn{6}{|c|}{ Painel B - Com exclusão de indicadores } \\
\hline Variáveis & $\begin{array}{l}\text { Alfa de } \\
\text { Cronbach }\end{array}$ & rho_A & $\begin{array}{l}\text { Confiabilidade } \\
\text { composta }\end{array}$ & $\mathbf{R}^{\mathbf{2}}$ & $\begin{array}{c}\text { Variância média } \\
\text { extraída (AVE) }\end{array}$ \\
\hline Estilos de tomada de decisão & 0,742 & 0,769 & 0,749 & $-0,127$ & 0,289 \\
\hline Flexibilidade cognitiva & 0,730 & 0,759 & 0,792 & 0,199 & 0,252 \\
\hline Traços de personalidade & 0,681 & 0,718 & 0,744 & 0,344 & 0,252 \\
\hline Desempenho da tarefa & 0,786 & 0,813 & 0,862 & 0,317 & 0,612 \\
\hline
\end{tabular}

Fonte: Dados da pesquisa.

No Painel A da Tabela 1, o modelo de mensuração apresentou inicialmente alguns valores abaixo da significância estatística mínima sugerida na literatura, isto é, alfa de Cronbach e confiabilidade composta com cargas $<0,7$ (Hair Jr. et. al, 2014), e valores de AVE $<0,50$ (Henseler, Ringle \& Sinkovics, 2009). De acordo com Bido e Silva (2019), é recomendável excluir indicadores com cargas mais baixas. Contudo, para preservar a validade de conteúdo dos constructos optou-se por excluir o menor número possível de indicadores, ainda que os valores de AVE não alcancem a significância estatística desejável.

No Painel B da Tabela 1, observa-se que o valor do alfa de Cronbach apresentou sensível melhora para estilos de tomada de decisão, mas não para traços de personalidade. Por sua vez, o valor de confiabilidade composta para estilos de tomada de decisão passou a apresentar significância estatística. No entanto, a eliminação de seis questões $(10,12,13,14,16$ e 19) do constructo flexibilidade cognitiva resultou em aumento insignificante dos valores AVE, isso não prejudica a confiabilidade do modelo, contudo é uma restrição.

Assim, adentrou-se na segunda etapa da análise estatística, em que foi considerado o cenário dos valores com a exclusão de seis indicadores. 


\subsection{Modelo Estrutural}

Na Tabela 2 apresentam-se os valores estatísticos dos dados analisados para o modelo estrutural, considerando as relações propostas nas hipóteses da pesquisa.

Tabela 2

\begin{tabular}{lcccccc} 
Estatística descritiva & & & & & \\
\hline & Hipóteses & VIF & Amostra original & Desvio Padrão & Valor t & Valor $\mathbf{p}$ \\
\hline $\begin{array}{l}\text { Estilos de Tomada de Decisão } \rightarrow \\
\text { Desempenho da Tarefa }\end{array}$ & $\mathrm{H} 1(-)$ & 1,286 & -0.127 & 0.061 & 2.082 & 0.037 \\
\hline $\begin{array}{l}\text { Flexibilidade Cognitiva } \rightarrow \\
\text { Desempenho da Tarefa }\end{array}$ & $\mathrm{H} 2(+)$ & 1,662 & 0.199 & 0.068 & 2.915 & 0.004 \\
\hline $\begin{array}{l}\text { Traços de Personalidade } \rightarrow \\
\text { Desempenho da Tarefa }\end{array}$ & $\mathrm{H} 1(+)$ & 1,749 & 0.344 & 0.078 & 4.422 & 0.000 \\
\hline
\end{tabular}

Legenda: Valores de $\mathrm{p} \leq 0,05 ;$ VIF: $\leq 2 ;$ Desvio padrão $\leq 5$

Fonte: Dados da pesquisa

Os resultados da estatística descritiva mostram números satisfatórios para a significância das relações analisadas, em que os valores de VIF, que demonstram a colinearidade dos dados, estão abaixo de 2 , o desvio padrão é considerado baixo e os valores p são significantes ao nível $\leq 0,05$. Observa-se ainda que estilos de tomada de decisão apresentam relação negativa com desempenho da tarefa, enquanto as demais relações foram positivas, conforme esperado.

\subsection{Discussão dos Resultados}

A literatura fornece suporte teórico para o constructo desempenho no trabalho ser analisado sob três domínios: desempenho da tarefa, desempenho contextual e comportamento contraproducente no trabalho (Sackett \& Lievens, 2008). No entanto, no presente estudo, o foco de análise recaiu sobre o desempenho da tarefa, ligado aos comportamentos que contribuem para a produção de um bem ou prestação de um serviço.

A hipótese $\mathrm{H}_{1}$ apresentou relação negativa entre estilos de tomada de decisão e desempenho da tarefa, com valor $\mathrm{p}=0,037$ e significante ao nível de 0,05 , o que leva à rejeição da hipótese. É possível que as características de subjetividade e individualidade (Harren, 1979) presentes na percepção das tarefas e na tomada de decisões (Sohail, 2013) sejam um fator de complexidade. Além disso, o fator autorregulação, ou seja, a capacidade de o indivíduo manter um padrão estável de decisões ao longo do tempo (Löbler et al., 2019; Thunholm, 2004), não mensurado neste estudo, pode contribuir para uma relação negativa.

Thunholm (2004) destaca a necessidade de mais investigações sobre a relação entre os estilos de tomada de decisão e o comportamento de tarefa em decisões reais de trabalho. No presente estudo, não é possível analisar esta relação, contudo, seus resultados denotam a atualidade dessa lacuna. No campo da perícia isso é potencializado, visto que o trabalho não é padronizado e cada perito estabelece a metodologia que melhor se adequa ao objeto da perícia, o que pode impactar no desempenho da tarefa.

A hipótese $\mathrm{H}_{2}$ foi confirmada para a amostra analisada, com valor $\mathrm{p}=0,004$, o que suporta $\mathrm{o}$ pressuposto de que a flexibilidade cognitiva se relaciona positivamente com o desempenho da tarefa de peritos contábeis judiciais. A necessidade dos peritos contábeis se adaptarem às mais diversas estratégias do trabalho pericial influencia o desempenho da tarefa.

Esta inferência encontra apoio em Martin e Rubin (1995), que enfatizam que a flexibilidade cognitiva é a capacidade da consciência individual para reconhecer opções e alternativas disponíveis, ter a disposição de ser flexível e adaptável e ter autoeficácia em ser flexível. Isso pode melhorar o processo decisório e levar o indivíduo a se adequar à tarefa (Laureiro-Martínez \& Brusoni, 2018). 
A hipótese $\mathrm{H}_{3}$ é aceita, já que apontou relação estatística positiva para a relação entre traços de personalidade e desenho da tarefa de peritos contábeis judiciais, com valor $\mathrm{p}=0,000$. Este resultado coaduna com Gridwicha, Kulwanich, Piromkam e Kwanmuangvanich (2020), que utilizaram a modelagem de equações estruturais e encontraram relação estatística positiva entre traços de personalidade e desempenho no trabalho.

\section{Considerações Finais}

Esse estudo analisou os efeitos dos estilos de tomada de decisão, da flexibilidade cognitiva e dos traços de personalidade no desempenho da tarefa de peritos contábeis judiciais. Os resultados permitem concluir que o desempenho da tarefa é uma mensuração complexa que pode, além da racionalidade econômica, ser explicada por variáveis comportamentais, como as consideradas neste estudo.

Além disso, ele contribui com a literatura ao mostrar que é preciso expandir o escopo de análises do desempenho da tarefa para além de abordagens positivistas, ou seja, não se pode presumir que um indivíduo melhore seu desempenho unicamente por comandos racionais de trabalho. Portanto, os resultados deste estudo abrem caminhos de investigação e incentivam a busca de novas abordagens e constructos para explicar os comportamentos relacionados ao desempenho da tarefa de indivíduos.

O estudo também apresenta contribuições práticas ao considerar, na pesquisa empírica, aspectos comportamentais que podem afetar o desempenho da tarefa de peritos contábeis judiciais. A observação de fatores comportamentais no desempenho da tarefa pode contribuir para a seleção e indicação de peritos aptos a apresentar relatórios periciais mais aderentes ao objeto da perícia e eficazes em promover o equilíbrio da justiça.

Diante disso, considerando as inferências de Frezatti (2020), ao citar o estudo de Sabadini, Sampaio e Koeller (2009) e enfatizar a importância do bem-estar que a pesquisa deve gerar na vida das pessoas, buscou-se neste estudo encontrar respostas comportamentais que ajudem a compreender as decisões e reduzir o viés no trabalho de peritos contábeis. Porém, parcimônia é necessária nas inferências, uma vez que o uso de medidas subjetivas pode levar os indivíduos a interpretarem as questões de forma diferente (Kahneman \& Krueger, 2006).

Nesse aspecto é importante também considerar as restrições do autorrelato (Skinner, 1978), já que não é possível identificar se o item escolhido pelo respondente representa de fato seu comportamento real ou um comportamento socialmente desejável (Tracey, 2016).

Outro viés de instrumentos de autorrelatos é a aquiescência, que é a tendência dos indivíduos escolherem itens sem analisar devidamente seu conteúdo (Zanon, Lessa \& Dellazzana-Zanon, 2018). Contudo, encontrar respostas definitivas em pesquisas pode não se concretizar em uma primeira comunicação científica (Frezatti, 2020), assim considera-se que este estudo não é um fim em si, mas um ponto de partida que pode vir a contribuir com pesquisas futuras.

Por fim, as análises realizadas não permitem sua generalização para todas as tarefas da área pericial, porém, essa restrição oportuniza outros estudos, que podem analisar o desempenho na execução de tarefas específicas e na relação entre as normas de perícia contábil, as escolhas metodológicas do perito e as expectativas do juízo, pois mesmo que o perito siga as diretrizes legais, seu laudo pode não ser suficiente para atender ao objeto da perícia. Ademais, pesquisas em profundidade, como de estudos de caso, ou experimentos podem representar importantes maneiras de avançar nas investigações sobre a temática. 


\section{Apêndice A - Instrumentos da Pesquisa}

Traços de Personalidade (Gosling, Rentfrom \& Swann, 2003)

Indique para cada uma das declarações que seguem até que ponto você concorda ou discorda que o par de características se aplica a você, considerando a escala de 1 (discordo totalmente) a 5 (concordo totalmente).

1. Eu me vejo como extrovertido, entusiasmado.

2. Eu me vejo como crítico, briguento.

3. Eu me vejo como confiável, autodisciplinado.

4. Eu me vejo como ansioso, facilmente me chateio.

5. Eu me vejo como aberto a novas experiências, complexas.

6. Eu me vejo como reservado, quieto.

7. Eu me vejo como simpático, caloroso.

8. Eu me vejo como desorganizado, descuidado.

9. Eu me vejo como calmo, emocionalmente estável.

10. Eu me vejo como convencional, não criativo.

Estilos de Tomada de Decisão (Scott \& Bruce, 1995; instrumento validado no Brasil por Löbler, Reis, Nishi \& Tagliapietra, 2019)

Indique nas assertivas que seguem, relativas à forma como o indivíduo toma decisões importantes, até que ponto você concorda ou discorda com cada uma delas, considerando a escala de 1 (discordo totalmente) a 5 (concordo totalmente).

1. Frequentemente eu preciso da ajuda de outras pessoas na tomada de decisões importantes.

2. Eu raramente tomo decisões importantes sem consultar outras pessoas.

3. Se eu tenho o apoio de outras pessoas, é mais fácil para eu tomar decisões importantes.

4. Eu utilizo o conselho de outras pessoas na tomada de decisões importantes.

5. Eu gosto de ter alguém para me orientar na direção certa, quando estou diante de decisões importantes.

6. Eu postergo a tomada de decisão, sempre que possível.

7. Muitas vezes eu adio a tomada de decisões importantes.

8. Eu geralmente tomo decisões importantes no último minuto.

9. Eu prorrogo a tomada de decisões, porque pensar nisso me faz ficar apreensivo.

10. Eu tomo decisões de uma forma lógica e sistemática.

11. A minha tomada de decisão requer uma reflexão cuidadosa.

12. Ao tomar uma decisão, considero várias opções em termos de um objetivo específico.

13. Eu analiso todas as minhas opções antes de tomar uma decisão.

14. Ao tomar decisões, eu confio em meu instinto.

15. Quando tomo decisões, costumo confiar em minha intuição.

16. Ao tomar uma decisão, eu confio em meus sentimentos e reações.

17. Eu geralmente tomo decisões rápidas.

18. Eu costumo tomar decisões no calor do momento.

19. Eu tomo decisões rápidas. 
Flexibilidade Cognitiva (Martin \& Hubin, 1995)

Indique para cada uma das declarações que seguem até que ponto você concorda ou discorda que elas representam suas crenças e sentimentos sobre seu próprio comportamento, considerando a escala de 1 (discordo totalmente) a 5 (concordo totalmente).

1. Eu posso comunicar uma ideia de muitas maneiras diferentes.

2. Eu evito situações novas e incomuns.

3. Eu sinto que nunca consigo tomar decisões.

4. Eu posso encontrar soluções viáveis para problemas aparentemente insolúveis.

5. Eu raramente tenho escolhas ao decidir como me comportar.

6. Eu estou disposto a trabalhar em soluções criativas para problemas.

7. Em qualquer situação, sou capaz de agir de maneira apropriada.

8. Meu comportamento é resultado de decisões conscientes que eu tomo.

9. Eu tenho muitas maneiras possíveis de me comportar em qualquer situação.

10. Eu tenho dificuldade em usar meu conhecimento sobre um determinado tópico em situações da vida real.

11. Eu estou disposto a ouvir e considerar alternativas para lidar com um problema.

12. Eu tenho a autoconfiança necessária para lidar com diferentes maneiras de me comportar.

Desempenho da Tarefa (Kathuria e Davis, 2001)

Indique para cada uma das situações que seguem o seu grau de satisfação com o seu desempenho no trabalho, considerando a escala de 1 (não satisfeito) a 5 (muito satisfeito)

1. Precisão do trabalho realizado.

2. Quantidade de trabalho realizado.

3. Qualidade do trabalho realizado.

4. Eficiência operacional.

\section{Referências}

Anderson, J.C., \& Gerbing, D.W. (1988). Structural equation modeling in practice: A review and recommended two-step approach. Psychological Bulletin, 103(3), pp. 411-423. Doi: https://doi. org/10.1037/0033-2909.103.3.411

Barrick, M.R., \& Mount, M.K. (2003). Select on conscientiousness and emotional stability. In: Locke, E. A. (Ed.). Handbook of principles of organizational behavior (2. ed.). Willey, New Jersey.

Benbasat, I., \& Dexter, A.S. (1982). Individual differences in the use of decision support aids. Journal of Accounting Research, 20(1), pp. 1-11. Doi: 10.2307/2490759

Bernstein, P.L. (1997). Desafio aos deuses: A fascinante história do risco (16. ed.). Campus, Rio de Janeiro.

Bendassolli, P.F. (2012). Desempenho no trabalho: revisão da literatura. Psicologia Argumento, 30(68), pp. 171-184. Doi: 10.7213/psicol.argum.5895

Birnberg, J.G., Luft, J., \& Shields, M.D. (2006). Psychology theory in management accounting research. In: Chapman, C.S.; Hopwood, A.G., \& Shields, M.D (Eds.). Handbooks of management accounting research (vol. 1), pp. 113-135. Elsevier, Oxford. Doi: https://doi.org/10.1016/S1751-3243(06)01004-2

Bromwich, M., \& Scapens, R.W. (2016). Management Accounting Research: 25 years on. Management Accounting Research, 25th Anniversary Conference, 31, pp. 1-9. Doi: http://dx.doi.org/10.1016/j.mar.2016.03.002

Butler, S.A., \& Ghosh, D. (2015). Individual differences in managerial accounting judgments and decision making. The British Accounting Review, 47(1), pp. 33-45. Doi: https://doi.org/10.1016/j.bar.2014.09.002 
Cagnin, S. (2009). Neuropsicologia cognitiva e psicologia cognitiva: o que o estudo da cognição deficitária pode nos dizer sobre o funcionamento cognitivo normal? Psicologia em Pesquisa, 3(1), pp. 16-30. Doi: https://doi.org/10.24879/200900300100392.

Capovilla, A.G.S., Assef, E.C.S., \& Cozza, H.F.P. (2007). Avaliação neuropsicológica das funções executivas e relação com desatenção e hiperatividade. Avaliação Psicológica, 6(1), pp. 51-60.

Cheng, M.M., Luckett, P.F., \& Schulz, A.K-D. (2003). The effects of cognitive style diversity on decisionmaking dyads: An empirical analysis in the context of a complex task. Behavioral Research in Accounting, 15(1), pp. 39-62. Doi: https://doi.org/10.2308/bria.2003.15.1.39

Chenhall, R., \& Morris, D. (1991). The effect of cognitive style and sponsorship bias on the treatment of opportunity costs in resource allocation decisions. Accounting, Organizations and Society, 16(1), pp. 27-46. Doi: https://doi.org/10.1016/0361-3682(91)90031-9

Chopik, W.J., \& Kitayama, S. (2017). Personality change across the life span: Insights from a cross-cultural, longitudinal study. Journal of Personality, 83(3), pp. 508-521. Doi: https://doi.org/10.1111/jopy.12332

Cubel, M., Nuevo-Chiquero, A., Sanchez-Pages, S., \& Vidal-Fernandez, M. (2016). Do personality traits affect productivity? Evidence from the laboratory. The Economic Journal, 126(592), pp. 654-681. Doi: https://doi.org/10.1111/ecoj.12373

Cunha, P.R., Silva, C.T., Peyerl, D.A., \& Haveroth, J. (2019). Influência dos traços de personalidade no ceticismo profissional de auditores independentes. Revista de Contabilidade e Organizações, 13(e158537), pp. 1-14. Doi: https://doi.org/10.11606/issn.1982-6486.rco.2019.158537

Debusscher, J., Hofmans, J., \& Fruit, F. (2016). From state neuroticism to momentary task performance: A person $\times$ situation approach. European Journal of Work and Organizational Psychology, 25(1), pp. 89-104. Doi: https://doi.org/10.1080/1359432X.2014.983085

Diógenes, S.B., \& Silva, D. (2019). Recursos e técnicas de ensino e pesquisa: Smart PLS 3 - especificação, estimação, avaliação e relato. Administração: Ensino e Pesquisa, 20(2), pp. 488-536. Doi: 10.13058/ raep.2019.v20n2.1545

França, J.A., \& Barbosa, A.B. (2015). O ensino de perícia contábil em Brasília: percepções dos estudantes de ciências contábeis. Revista Catarinense da Ciência Contábil, 14(43), pp. 63-73. Doi: http://dx.doi. org/10.16930/2237-7662/rccc.v14n43p63-73

Frankovsky, M., Birknerova, Z., \& Zbihlejova, L. (2016). Assessment of occurrence predictors of cognitive distortions in managerial decisions. Polish Journal of Management Studies, 14(2), pp. 61-70. Doi: 10.17512/pjms.2016.14.2.06

Frezatti, F. (2020). Pentágono da qualidade na publicação acadêmica. Revista de Educação e Pesquisa em Contabilidade - REPEC, 14(4), pp. 422-426. Doi: http://dx.doi.org/10.17524/repec.v14i4.2802

Fuller, L.R., \& Kaplan, S.E. (2004). A Note about the effect of auditor cognitive style on task performance. Behavioral Research in Accounting, 16(1), pp. 131-143. Doi: https://doi.org/10.2308/ bria.2004.16.1.131

Ge, W., Matsumoto, D., \& Zhang, J.L. (2011). Do CFOs have style? An empirical investigation of the effect of individual CFOs on accounting practices. Contemporary Accounting Research, 28(4), pp. 11411179. Doi: https://doi.org/10.1111/j.1911-3846.2011.01097.x

George, J.M., \& Zhou, J. (2007). Dual tuning in a supportive context: Joint contributions of positive mood, negative mood, and supervisory behaviors to employee creativity. Academy of Management Journal, 50(3), pp. 605-622. Doi: 10.5465/AMJ.2007.25525934

Goldberg, L.R. (1992). The development of markers for the Big-Five factor structure. Psychological Assessment, 4(1), pp. 26-42. Doi: https://doi.org/10.1037/1040-3590.4.1.26 
Gosling, S.D., Rentfrom, P.J., \& Swann, W.B.J. (2003). A very brief measure of the big-five personality traits. Journal of Research in Personality, 37(6), pp. 504-528. Doi: doi:10.1016/S0092-6566(03)00046-1

Gridwicha, P., Kulwanich, A, Piromkam, B., \& Kwanmuangvanich, P. (2020). Role of personality traits on employees job performance in pharmaceutical industry in Thailand. Sys Rev Pharm, 11(3), pp. 185-194. Recuperado de: http://sysrevpharm.org/fulltext/196-1584097466.pdf

Guerra, M.C.A.G. (2013). Flexibilidade cognitiva: estudos de conceptualização e operacionalização do construto. Tese de Doutorado, Universidade de Évora, Portugal-PT. Recuperado de: http://dspace. uevora.pt/rdpc/handle/10174/10867

Guerra, C.G, Candeias, A., \& Prieto, G. (2014). Flexibilidade cognitiva: repensar o conceito e a medida da inteligência. I Seminário Internacional de Cognição, Aprendizagem e Rendimento. Universidade do Minho, Portugal.

Hair Jr., J.F., Hult, T.M., Ringle, C.M., \& Sarstedt, M. (2014). A primer on partial least squares structural equation modeling (PLS-SEM). Sage, Los Angeles.

Harren, V.A. (1979). A model of career decision making for college students. Journal of Vocational Behavior, 14(2), pp. 119-133. Doi: https://doi.org/10.1016/0001-8791(79)90065-4

Hastie, R. (2001). Problems for judgment and decision making. Annual Review of Psychology, 52, pp. 653683. Doi: https://doi.org/10.1146/annurev.psych.52.1.653

Henseler, J., Ringle, C.M., \& Sinkovics, R.R. (2009). The use of partial least squares path modeling in international marketing. In: Sinkovics, R.R., \& Ghauri, P.N. (Eds.). New challenges to international marketing (vol. 20), pp. 277-319. Emerald, Bingley. Doi: https://doi.org/10.1108/S14747979(2009)0000020014

Hoog, W.A.Z. (2008). Prova pericial contábil: aspectos práticos \& fundamentais (5.ed.). Juruá, Curitiba.

Hudson, N.W., \& Fraley, R.C. (2015). Volitional personality trait change: Can people choose to change their personality traits? Journal of Personality and Social Psychology, 109(3), pp. 490-507. Doi: http:// dx.doi.org/10.1037/pspp0000021

Kahneman, D., \& Krueger, A.B. (2006). Developments in the measurement of subjective well-being. Journal of Economics Perspectives, 20(1), pp. 3-24. Doi: 10.1257/089533006776526030

Kahneman, D., \& Tversky, A. (2012). Rápido e devagar: Duas formas de pensar. Objetiva, Rio de Janeiro.

Kathuria, R., \& Davis, E.B. (2001). Quality and work force management practices: the managerial performance implication. Production and Operations Management, 10(4), pp. 460-477. Doi: https:// doi.org/10.1111/j.1937-5956.2001.tb00087.x

Laureiro-Martínez, D., \& Brusoni, S. (2018). Flexibilidade cognitiva e tomada de decisão adaptativa: evidências de um estudo de laboratório de tomadores de decisão especializados. Strategic Management Journal, 39(4), pp. 1031-1058. Doi: 10.1002 / smj.2774

Lauriola, M., \& Levin, I.P. (2001). Personality traits and risky decision-making in a controlled experimental task: an exploratory study. Personality and Individual Differences, 31(2), pp. 215-226. Doi: https:// doi.org/10.1016/S0191-8869(00)00130-6

Lima Filho, R.N., Bruni, A.L., Sampaio, M.S., Cordeiro Filho, J.B., \& Carvalho Jr., C.V.O. (2010). Heurísticas e práticas orçamentárias: um estudo experimental. Sociedade, Contabilidade e Gestão, 5(1), 42-58.

Lonescu, T. (2012). Exploring the nature of cognitive flexibility. New Ideas in Psychology, 30(2), pp. 190200. Doi: https://doi.org/10.1016/j.newideapsych.2011.11.001

Lucena, W.G.L., Fernandes, M.S.A., \& Silva, J.D.G. (2011). A contabilidade comportamental e os efeitos cognitivos no processo decisório: uma amostra de operadores de contabilidade. Revista Universo Contábil, 7(3), pp. 41-58. Doi: 10.4270/ruc.2011321 
Luft, J., Shields, M.D., \& Thomas, T.F. (2016). Additional information in accounting reports: Effects on management decisions and subjective performance evaluations under causal ambiguity. Contemporary Accounting Research, 33(2), pp. 526-550. Doi: https://doi.org/10.1111/1911-3846.12156

Löbler, M.L., Reis, E.; Nishi, J.M., \& Tagliapietra, R.D. (2019). Inventário de estilos de tomada de decisão: Validação de instrumento no contexto brasileiro. Revista de Administração - UNIMEP, 17(1), pp. 214-236. Recuperado em 31 de maio de 2021 de: http://www.raunimep.com.br/ojs/index.php/rau/ article/view/1167/785

Macêdo, A.F.P. (2016). Impacto dos traços da personalidade, vieses cognitivos e características sociodemográficas no perfil de risco de pessoas físicas participantes de leilões no Brasil. Tese de Doutorado, Universidade do Minho, Braga, Portugal.

Mahama, H., \& Cheng, M.M. (2013). The effect of managers' enabling perceptions on costing system use, psychological empowerment, and task Performance. Behavioral Research in Accounting, 25(1), pp. 89-114. Doi: https://doi.org/10.2308/bria-50333

Malkiel, B.G., Fama, E.F. (1970). Efficient capital markets a review of theory and empirical work. The Journal of Finance, 25(2), pp. 383-417. Doi: https://doi.org/10.1111/j.1540-6261.1970.tb00518.x

Marras, J.P. (2011). Administração de recursos humanos. Futura, São Paulo.

Martin, M.M., \& Rubin, R.B. (1995). A new measure of cognitive flexibility. Psychological Reports, 76(2), pp. 623-626. Doi: https://doi.org/10.2466/pr0.1995.76.2.623

Martínez, D.L., \& Brusoni, S. (2018). Cognitive flexibility and adaptive decision-making: evidence from a laboratory study of expert decision makers. Strategic Management, 39(4), pp. 1031-1058. Doi: https://doi.org/10.1002/smj.2774

Melo, T.M., \& Fucidji, J.R. (2016). Racionalidade limitada e a tomada de decisão em sistemas complexos. Revista de Economia Política, 36(3), pp. 622-645. Doi: https://doi.org/10.1590/010131572016v36n03a09

Mendonça Neto, O.R.M., Cardoso, R.L., Oyadomari, J.C.P., \& Silva, D. (2009). O Processo decisório em ambientes contábeis: Uma aplicação da teoria dos modelos mentais probabilísticos. Contabilidade Vista \& Revista, 20(4), pp. 109-130. Recuperado em 31 de maio de 2021 de: https://revistas.face. ufmg.br/index.php/contabilidadevistaerevista/article/view/718

Messick, S. (1984). The nature of cognitive styles: problems and promise in educational practice. Educational Psychologist, 19(2), pp. 59-74. Doi: https://doi.org/10.1080/00461528409529283

Monzani, L., Ripoll, P., \& Peiró, J.M. (2015). The moderator role of followers' personality traits in the relations between leadership styles, two types of task performance and work result satisfaction. European Journal of Work and Organizational Psychology, 24(3), pp. 444-461. Doi: https://doi.or g/10.1080/1359432X.2014.911173

Motowildo, S.J., Borman, W.C., \& Schmit, M.J. (2013). A theory of individual differences in task and contextual performance. Human Performance, 10(2), pp. 71-83. Doi: http://dx.doi.org/10.1207/ s15327043hup1002_1

Mueller, B.A., \& Shepherd, D.A. (2016). Making the most of failure experiences: Exploring the relationship between business failure and the identification of business opportunities. Entrepreneurship Theory and Practice, 40(3), 457-487. Doi: https://doi.org/10.1111/etap.12116

Murro, E.V.B., \& Beuren, I.M. Redes de atores na perícia contábil judicial: uma análise à luz da Teoria AtorRede. Revista Brasileira de Gestão de Negócios, 18(62), pp. 633-657. Doi: 10.7819/rbgn.v18i62.2743

Neufeld, C.B., Brust, P.G., \& Stein, L.M. (2011). Bases epistemológicas da psicologia cognitiva experimental. Psicologia: Teoria e Pesquisa, 27(1), pp. 103-112. Doi: https://doi.org/10.1590/S010237722011000100013 
Neumann, J.V., \& Morgenstern, O. (1944). Theory of games and economic behavior. The Review of Economics and Statistics, 29(1), pp. 47-52.

Oblak, L., Licen, M., \& Slapnicar, S. (2018). The role of cognitive frames in combined decisions about risk and effort. Management Accounting Research, 39(3), pp. 35-46. Doi: https://doi.org/10.1016/j. mar.2017.07.001

Pereira, B.A.D., Löbler, M. L., \& Simonetto, E.O. (2010). Análise dos modelos de tomada de decisão sob o enfoque cognitivo. Revista Administração UFSM, 3(2), pp. 260-268. Recuperado em 1/06/2021 em: http://www.spell.org.br/documentos/ver/5140/analise-dos-modelos-de-tomada-decisao-sobo-enfoque-cognitivo/i/pt-br

Pereira, I.V., Silva, C.A.T., \& Tavares Jr. E.P. (2017). Comportamento decisório dos contadores sob a perspectiva da Teoria dos Prospectos. Revista Ambiente Contábil, 9(2), pp. 222-239. Recuperado em 1/06/2021 em: https://periodicos.ufrn.br/ambiente/article/view/11128

Perez, G., Valero, S., Cesar, A.M.R.V.C., \& Medeiros Júnior, A. (2017). Tomada de decisão em metas orçamentárias: o estudo de uma empresa do setor siderúrgico baseado no modelo cognitivo. Revista FAE, 20(1), pp. 69-92. Recuperado em 1/06/2021 em: https://revistafae.fae.edu/revistafae/article/view/102

Plous, S. (1993). The psychology of judgment and decision making. McGraw-Hill Education, New York.

Riding, R.J. (1997). On the nature of cognitive style. Educational Psychology, 17(1-2), pp. 29-49. Doi: https://doi.org/10.1080/0144341970170102

Sabadini, A.A.Z.P., Sampaio, M.I.C., \& Koeller, S.H. (2009). Publicar em psicologia. (A. B. de E. C. de Psicologia, Ed.). São Paulo: Instituto de Psicologia de São Paulo.

Sackett, P.R., \& Lievens, F. (2008). Personnel selection. Annual Review of Psychology, 59, pp. 419-450. Doi: https://doi.org/10.1146/annurev.psych.59.103006.093716

Santos, J.O., \& Barros, C.A.S. (2011). O que determina a tomada de decisão financeira: razão ou emoção? Revista Brasileira de Gestão de Negócios, 13(38), pp. 7-20. Recuperado em 1/06/2021: http://www. spell.org.br/documentos/ver/6619/o-que-determina-a-tomada-de-decisao-financeira--razao-ouemocao-/i/pt-br

Santos, A.P.A., Sisto, F.F., \& Martins, R.M.M. (2003). Estilos cognitivos e personalidade: um estudo exploratório de evidências de validade. Psico-USF, 8(1), pp. 11-19. Doi: https://doi.org/10.1590/ S1413-82712003000100003.

Saucier, G. (1994). Mini-markers: a brief version of goldberg's unipolar big-five markers. Journal of Personality Assessment, 63(3), pp. 506-516. Doi: https://doi.org/10.1207/s15327752jpa6303_8

Scott, S.G., \& Bruce, R.A. (1995). Decision-making style: the development and assessment of a new measure. Educational and Psychological Measurement, 55(5), pp. 818-831. Doi: https://doi. org/10.1177/0013164495055005017

Simon, H.A. (1955). A behavioral model of rational choice. Quartely Journal of Economics, 69(1), pp. 99118. Doi: https://doi.org/10.2307/1884852

Skinner, B. F. (1978). O comportamento verbal. São Paulo: Cultrix.

Sohail, T. (2013). Decision making style of women university teachers. J.R.S.P., 50(2). Recuperado de: http://pu.edu.pk/images/journal/history/PDF-FILES/ARTICLE\%208\%20TALAT\%20190-205_v50_ no2_2013.pdf

Spiro, R., Vispoel, W., Schmitz, J.G., Samarapungavan, A., \& Boerger, A.E. (1987). Knowledge aquisition for application: cognitive flexibility and transfer in complex content domains. In: Britton, B. C., \& Glynn, S. M. (eds.). Executive control in processes in reading, pp. 177-199. Lawrence Erlbaum Associates, New Jersey. 
Tracey, T.J.G. (2016). A note on socially desirable responding. Journal of Counseling Psychology, 63(2), pp. 224-232. Doi: https://doi.org/10.1037/cou0000135.

Trentini, C.M., Hutz, C.S., Bandeira, D.R., Teixeira, M.A.P., Gonçalves, M.T.A., \& Thomazoni, A.R. (2009). Correlações entre a EFN - Escala Fatorial de Neuroticismo e o IFP - Inventário Fatorial de Personalidade. Avaliação Psicológica, 8(2), pp. 209-217. Recuperado em 1/06/2021 de: http://pepsic. bvsalud.org/scielo.php?script=sci_arttext\&pid=S1677-04712009000200007

Thunholm, P. (2004). Decision-making style: Habit, style or both? Personality and Individual Differences, 36(4), pp. 931-944. Doi: https://doi.org/10.1016/S0191-8869(03)00162-4

Weick, K.E. (1995). Sensemaking in organizations. Sage, Thousand Oaks.

Zanon, C., Lessa, J.P.A. \& Dellazzana-Zanon, L.V. (2018). Aquiescência em autorrelatos de personalidade: uma comparação de métodos. Avaliação Psicológica, 17(4), pp. 428-438. Doi: http://dx.doi. org/10.15689/ap.2018.1704.3.03 